\title{
Comparative genomic analysis of mycobacteriophage Tweety: evolutionary insights and construction of compatible site-specific integration vectors for mycobacteria
}

\begin{abstract}
Correspondence
Graham F. Hatfull gfh@pitt.edu
\end{abstract}

Received 10 April 2007

Revised 28 April 2007

Accepted 1 May 2007

\author{
Thuy T. Pham, ${ }^{1}$ Deborah Jacobs-Sera, ${ }^{1}$ Marisa L. Pedulla, ${ }^{2}$ \\ Roger W. Hendrix ${ }^{1}$ and Graham F. Hatfull ${ }^{1}$ \\ ${ }^{1}$ Department of Biological Sciences and Pittsburgh Bacteriophage Institute, \\ University of Pittsburgh, Pittsburgh, PA 15260, USA \\ ${ }^{2}$ Department of Biology, Montana Tech, University of Montana, Butte, MT 59701, USA
}

\section{INTRODUCTION}

The isolation and comparative genomic analysis of 30 mycobacteriophages (viruses that infect mycobacterial hosts) reveals them to have high genetic diversity and to typically contain genomes that are genetic mosaics with modules shared with other phage genomes (Hatfull et al., 2006; Pedulla et al., 2003). Nucleotide sequence comparison of these genomes shows that while they are overall highly diverse, there are several smaller clusters within the group with genomes that are more similar to each other than to other mycobacteriophage genomes. Similar clusters

\footnotetext{
Abbreviation: BCG, bacille Calmette-Guérin.

The GenBank/EMBL/DDBJ accession number for the genome sequence of mycobacteriophage Tweety is EF536069.

Two supplementary figures showing the alignment of Tweety genes 20 and 21 with Che8 gene 2 and confirming a nucleotide deletion in the Tweety genome relative to the homologous segment in phage Llij, a larger version of Fig. 3, and two supplementary tables listing nucleotide repeats in Tweety gene 54 and repeated sequences in phages Tweety gp54, Che8 gp57 and PMC gp51 are available with the online version of this paper.
}

were revealed through a metaproteomic analysis in which all 3350 putative protein products were organized into related 'phamilies' and the genomes compared according to the presence or absence of phamily members (Hatfull et al., 2006). The availability of groups of closely related phages in the context of a larger, more diverse, group significantly enhances the power of comparative genomic analyses. In particular, comparisons among closely related sequences have the potential to reveal the nature of individual mutational steps of phage evolution, unconfounded by multiple overlapping events.

Characterization of mycobacteriophage genomes not only provides insights into viral diversity and evolution but also offers a large, diverse and complex toolbox from which a variety of applications useful for mycobacterial genetics can be derived. A recent example is the identification of mycobacteriophage genes encoding recombination functions related to RecE and RecT which, while rare among mycobacteriophages, are both found in phage Che9c (van Kessel \& Hatfull, 2007). These have been utilized to develop a recombineering system to facilitate the construction of gene-replacement mutants by allelic exchange 
in both Mycobacterium smegmatis and Mycobacterium tuberculosis (van Kessel \& Hatfull, 2007). Other examples include the use of phage immunity loci as genetically selectable markers (Donnelly-Wu et al., 1993; Jain \& Hatfull, 2000), regulated gene expression systems (Brown et al., 1997), and exploitation of phage integration systems (Lee et al., 1991).

The construction of integration-proficient plasmid vectors based on the integration system of mycobacteriophage L5 enables the simple insertion of genes into the chromosomes of both fast- and slow-growing mycobacteria (Lee et al., 1991; Stover et al., 1991) and similar vectors based on other phages such as Ms6 have been described previously (Freitas-Vieira et al., 1998). Provided that the phageencoded recombination directionality factor (RDF) (Lewis \& Hatfull, 2001) is not present in these vectors then the integrated DNAs are more stably maintained in the absence of selection than extrachromosomal plasmid vectors; however, excision-independent integrase-mediated excisive recombination can lead to plasmid loss and accumulation of excised derivatives, especially when the integrated sequences express products deleterious to growth of the recombinant (Springer et al., 2001). These events can be avoided by using transient expression of integrase to construct recombinants such that the int gene is not present in the stably transformed strains (Hatfull, 2004; Peña et al., 1997). Introduction of the L5 excise (gene 36) into integrated recombinants leads to efficient integrasemediated excision (Lewis \& Hatfull, 2000) and this has been exploited to determine whether genes are essential for mycobacterial growth (Parish et al., 2001) and to switch integrated plasmid copies (Pashley \& Parish, 2003).

A primary benefit of these integration-proficient vector systems is that they enable the construction of single-copy recombinants that avoid the phenotypic effects of multicopy recombinants, including phage and drug resistance (Banerjee et al., 1994; Barsom \& Hatfull, 1996). However, there are often genetic applications that require the insertion of more than one element into the chromosome and methods have been described to piggy-back multiple insertions using L5 integration vectors (Saviola \& Bishai, 2004), to manipulate Ms6 vectors to confer integration at different chromosomal loci (Vultos et al., 2006), or to use alternative integration systems such as those derived from serine-integrases $\phi$ Rv1 and Bxb1 (Bibb et al., 2005; Kim et al., 2003). However, these have significant limitations, including reduced frequency, limited strain utilization, or, in the case of the serine-integrases, interruption of chromosomal genes (Kim et al., 2003; Ojha et al., 2005). There is thus a need for additional integration-proficient vectors that are fully compatible with other vector systems.

In this paper we describe the isolation and genomic characterization of mycobacteriophage Tweety and the development of integration-proficient plasmids carrying the Tweety attP-int region that efficiently transform both fast- and slow-growing mycobacteria. These Tweety-derived vectors integrate at a tRNA ${ }^{\text {Lys }}$ gene as distinct from the tRNA ${ }^{\text {Gly }}$ chromosomal locus used by L5-derived vectors and are fully compatible, such that co-transformants with both integrating vector systems can be recovered from a single electroporation. $M$. smegmatis recombinants derived by Tweety-mediated integration are more stably maintained than recombinants derived using L5 integration-proficient vectors in the presence of their cognate integrases and should prove to be useful additions to the arsenal of tools available for mycobacterial genetic manipulation.

\section{METHODS}

Bacterial strains. Mycobacterium smegmatis $\mathrm{mc}^{2} 155$ and Mycobacterium bovis bacille Calmette-Guérin (BCG) have been described previously (Jacobs et al., 1991; Snapper et al., 1990); electrocompetent cells were prepared as described previously (Bibb \& Hatfull, 2002) and transformed using 0.05-1 $\mu \mathrm{g}$ DNA. Media were supplemented with carbenicillin $\left(50 \mu \mathrm{g} \mathrm{ml}^{-1}\right)$, cycloheximide $\left(10 \mu \mathrm{g} \mathrm{ml}^{-1}\right)$, kanamycin $\left(20 \mu \mathrm{g} \mathrm{ml}^{-1}\right)$, hygromycin $\left(50 \mu \mathrm{g} \mathrm{ml}^{-1}\right)$ or gentamicin $\left(10 \mu \mathrm{g} \mathrm{ml}^{-1}\right)$ as required.

Phage isolation and genome sequencing. Tweety was isolated from a moist soil sample from a lawn in the Oakland district near the University of Pittsburgh (PA, USA) . Tweety was plaque purified and sequenced using a shotgun approach as described previously (Pedulla et al., 2003; Sarkis \& Hatfull, 1998). The GenBank accession number is EF536069.

\begin{abstract}
Plasmids and DNA. Plasmid pMH94 is an L5 integration-proficient vector that has been described previously (Lee et al., 1991). Plasmids pJV39 and pJV44 were kind gifts from Julia van Kessel, University of Pittsburgh. Plasmid pJV39 is similar to pMH94 but confers hygromycin resistance $\left(\mathrm{Hyg}^{\mathrm{R}}\right)$ instead of kanamycin resistance $\left(\mathrm{Kan}^{\mathrm{R}}\right)$. Plasmids pTTP1A and pTTP1B were constructed as follows. Two primers with XhoI restriction sites were designed and used to amplify the attP and int region from Tweety genomic DNA. This $1.7 \mathrm{~kb}$ fragment was inserted by blunt-end cloning into vector pMOSBlue. A clone containing Tweety attP and int was identified and digested with XhoI, and the fragment was subcloned into SalIdigested pMH94. Both pTTP1A and pTTP1B contain the Tweety attP and int, oriE, and kanamycin- and ampicillin-resistance genes. Plasmids pTTP1A and pTTP1B differ in regard to the orientation of the attP-int region with respect to the plasmid backbone. DNA manipulations and agarose gel electrophoresis were as described by Sambrook et al. (1989).
\end{abstract}

PCR assays. Site-specific integration between the homologous sequences of Tweety attP and $M$. smegmatis attB was confirmed using PCR assays. Transformants were prepared for colony PCR by suspending in $200 \mu \mathrm{l} \mathrm{H}_{2} \mathrm{O}$, vortexing 20 times, and heating at $95{ }^{\circ} \mathrm{C}$ for $5 \mathrm{~min}$. Approximately $5 \mu \mathrm{l}$ of the colony mix was used along with $P f u$ polymerase (Stratagene), dNTPs $(10 \mathrm{mM})$ and $5 \%(\mathrm{v} / \mathrm{v})$ DMSO. The four primers used to amplify the attL and attR regions of the recombinant chromosome were TTP1a (5'-CAGTCACGACGTTGTAAAACGACGG-3'), TTP1b (5'-GTCACCGAAAGGCGTGCCCTTGTC- $\left.3^{\prime}\right)$, TTP1d (5'-GACCGCTTCAAGAGCGAGCAGTAC- $\left.3^{\prime}\right)$ and TTP1e (5'-TCCCGTTGAATATGGCTCATAACACCC-3'). PCR products were analysed by gel electrophoresis.

Plasmid stability. Transformants of M. smegmatis derived from pTTP1A and pTTP1B were inoculated into Middlebrook 7H10 medium containing ADC [albumin $\left(5 \mathrm{~g} \mathrm{l}^{-1}\right)$, dextrose $\left(2 \mathrm{~g} \mathrm{l}^{-1}\right), \mathrm{NaCl}$ 
$\left(0.85 \mathrm{~g} \mathrm{l}^{-1}\right)$ ], Tween $80(0.05 \%)$ and kanamycin $\left(20 \mu \mathrm{g} \mathrm{ml}^{-1}\right)$ and grown to saturation. Cultures were diluted 1:10000 into antibioticfree medium and allowed to grow back to saturation (Lee et al., 1991). The cultures were repeatedly diluted and grown for a total of approximately 35 generations. Cell samples were then plated for single colonies on solid 7H10/ADC medium in the presence and absence of kanamycin to determine the proportion of antibiotic-resistant colonies.

Electron microscopy. A suspension of CsCl-purified virions was applied to a sample grid with a carbon-coated nitrocellulose film, stained with $2 \%$ uranyl acetate, and examined in a FEI Morgagni 268 transmission electron microscope equipped with an AMT digital camera system.

\section{RESULTS}

\section{Isolation and genomic sequencing of mycobacteriophage Tweety}

Mycobacteriophage Tweety was isolated from a soil sample from the Oakland district of Pittsburgh, and was identified (without amplification) as a p.f.u. on a lawn of $M$. smegmatis $\mathrm{mc}^{2} 155$. Following plaque purification, hightitre stocks were prepared and Tweety virions were examined by electron microscopy. Tweety particles have a morphology typical of the Siphoviridae, with an isometric head approximately $60 \mathrm{~nm}$ in diameter and a flexible tail approximately $175 \mathrm{~nm}$ long (Fig. 1); this morphology is the most common one found among mycobacteriophages (Hatfull et al., 2006). A small, possibly double-layered, baseplate structure is visible at the tip of the tail, with some apparently flexible fibrous structures extending beyond it. Tweety forms lightly turbid plaques on lawns of $M$.

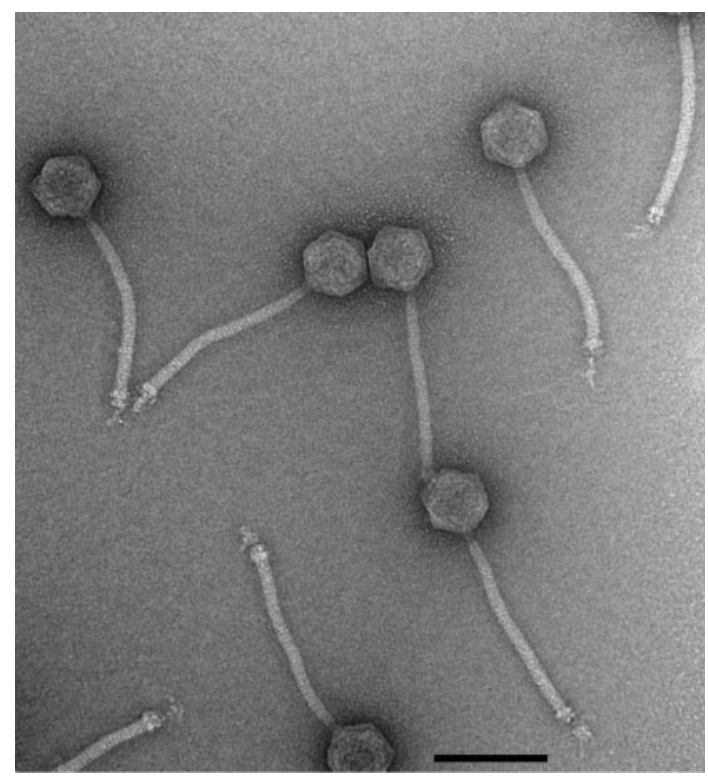

Fig. 1. Virion morphology of Tweety. Electron micrograph of mycobacteriophage Tweety particles. Scale bar, $100 \mathrm{~nm}$. smegmatis, a plaque morphology that is extremely common among mycobacteriophages.

Preliminary restriction analysis of Tweety dsDNA from virions indicated that it is distinct from all previously characterized mycobacteriophages, and its complete genomic sequence was determined using a shotgun strategy. Tweety virion DNA is 58692 bp in length; it has unique ends with 10 base, single-stranded cohesive $3^{\prime}$-extensions (left end; 3'-GCCTTCCGCG). The Tweety genome is $61.7 \mathrm{~mol} \% \mathrm{G}+\mathrm{C}$, similar to other mycobacteriophage and mycobacterial genomes. Nucleotide sequence comparison with the 30 previously sequenced mycobacteriophage genomes revealed significant sequence similarity with mycobacteriophages Che8, Llij, PMC and, to a lesser degree, Che9d (Fig. 2). The extent of sequence similarity between Tweety and Che8, Llij and PMC appears to be highest in the leftmost parts of these genomes, while being weaker and discontinuous towards the rightmost parts.

\section{Organization of the Tweety genome}

Analysis of the Tweety genome reveals 109 potential ORFs (Table 1), all except eight of which are transcribed in the rightwards direction (Fig. 3). The overall genome organization shares similarities with other mycobacteriophages such as PMC, Llij and Che8 and differs from phages such as L5, D29 and their near relatives, in which genes in the right half of the genome are transcribed leftwards (Ford et al., 1998; Hatfull \& Sarkis, 1993; Hatfull et al., 2006; Pedulla et al., 2003). Most of the Tweety genome is utilized as protein-coding regions, although there are small noncoding regions between gene 109 and the right terminus, and between genes 42 and 43 , and genes 44 and 45 . We have not identified any tRNA, transfer-messenger RNA (tmRNA) or other small RNA genes. An integrase gene (43) of the tyrosine recombinase family lies close to the centre of the genome, and the left arm (genes 1-42) is very similar in organization and sequence to the corresponding parts of the Che8, Llij and PMC genomes (Fig. 3), with the main differences at the right end of the left arm. A putative stem-loop terminator for rightwards transcription is positioned at coordinates 33784-33827 immediately following the integrase gene.

\section{The Tweety genomic left arm: virion structure and assembly genes}

Many of the Tweety left arm genes are probably involved in virion structure and assembly, and genes 2, 3, 11 and 14 encode putative terminase, portal, major tail subunit and tapemeasure functions respectively, based on sequence similarity to proteins with established functions. Genes 15 , $18,19,21,24$ and 25 may all encode minor tail proteins, and we note that the gp19 sequence suggests a carboxypeptidase function, as seen also in several other mycobacteriophage genomes. The two ORFs (12 and 13) between the major tail subunit (11) and tapemeasure genes (14) are 


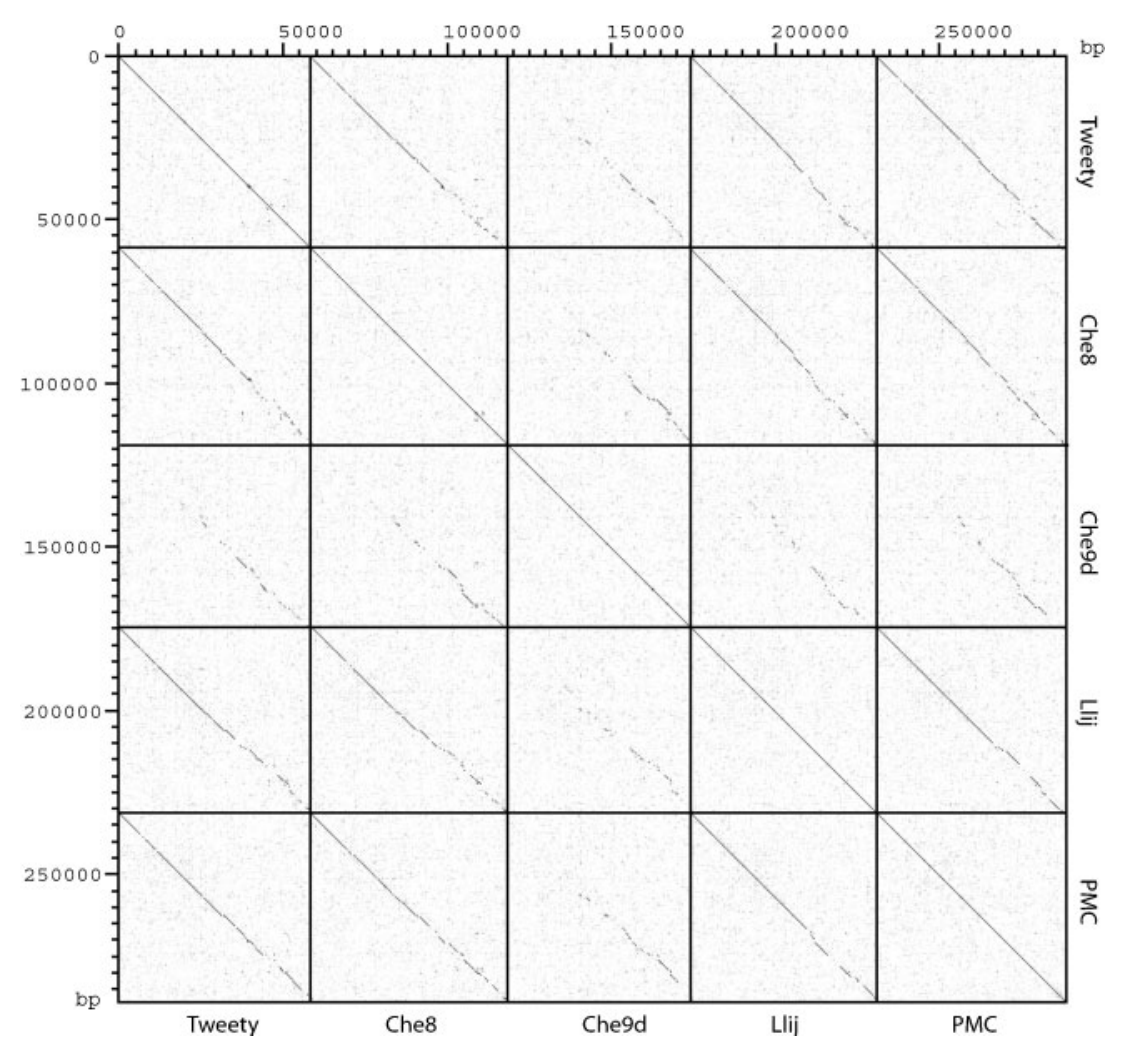

Fig. 2. Nucleotide sequence comparisons of the Tweety, Che8, Che9d, Llij and PMC genomes. The extent of DNA sequence similarity among these mycobacteriophage genomes is illustrated in a Dotter plot using a sliding window of $25 \mathrm{bp}$ (Sonnhammer \& Durbin, 1995). arranged such as to express the product of gene 12 (gp12) and a larger protein putatively generated via a translational -2 frameshift approximately $50 \mathrm{bp}$ from the end of gene 12. By analogy with phage lambda, the gp12 and gp12/13 products are probably involved as chaperones in tail assembly; the programmed frameshift is one of the bestconserved features of dsDNA tailed phages ( $\mathrm{Xu}$ et al., 2004). The tapemeasure gene is so named because the size of the encoded protein determines the length of the tail (Katsura \& Hendrix, 1984; Pedulla et al., 2003). In most cases the proportionality constant relating the two is $0.15 \mathrm{~nm}$ tail length per amino acid of tapemeasure protein, corresponding to an $\alpha$-helical structure for the tapemeasure protein. The measured length of the Tweety tail is $175 \mathrm{~nm}$ (above), and the 1176 amino acids of the tapemeasure protein would make an $\alpha$-helix of about $176 \mathrm{~nm}$, agreeing very closely with prediction.

The major capsid subunit is likely to be encoded by gene 6 , since we previously showed (unpublished observations) that the Che8 major capsid subunit is Che 8 gp6, which is $99 \%$ identical to Tweety gp6. When the sequence databases were searched with the Tweety gp6 sequence using the PSIBLAST algorithm, more than 100 phage capsid proteins were found, most with very low levels of similarity. Interestingly, after the near-perfect matches of Llij, PMC and Che8, the best matches are to the major capsid proteins of Escherichia coli phage T7 and its relatives, with some other mycobacteriophage capsid proteins farther down the list. The Tweety lysis genes $(30-32)$ are located at the right end of the left arm and encode lysin A (gp30), lysin B (gp31) and holin (gp32) functions respectively. Tweety gp35 has weak but significant similarity ( $25 \%$ identity, E-value, $10^{-5}$ ) to a putative DNA polymerase III $\varepsilon$ subunit of Xanthomonas phage OP1, and the position of a DNA metabolism gene in the left arm is an unusual feature (also found in phages Che8 and Llij). Mycobacteriophage Cjw1 encodes a homologue of Tweety gp35 (Cjw1 gp115), although in this genome it is located at the right end of the right arm (Pedulla et al., 2003).

The Tweety left arm encodes seven proteins (gp15, gp18, gp19, gp20, gp21, gp24 and gp25) that are all part of an extremely large phamily of minor tail proteins that have complex sequence relationships. Tweety gp18 is nearly identical throughout its entire length to Llij gp18 and PMC gp18, but the similar gene in Che8 encodes two proteins gp18 and gp19. A notable departure of the Tweety left arm from its Che8, Llij and PMC relatives is the apparent splitting of the Llij 20, Che 821 and PMC 20 into Tweety genes 20 and 21 (Fig. 3). The DNA sequences of these genes are very closely related although Tweety contains a 1 base deletion at codon 66 that shortens the ORF (see Supplementary Fig. S1, available with the online version of this paper); Tweety gene 21 corresponds to the 3 ' end of this segment, although it has a somewhat poor ribosomebinding site and it is uncertain whether it is likely to be expressed. The deletion does not appear to result from a sequencing error (Supplementary Fig. S2) and thus probably corresponds to a genomic change with specific 
Table 1. Coordinates and putative functions of Tweety genes

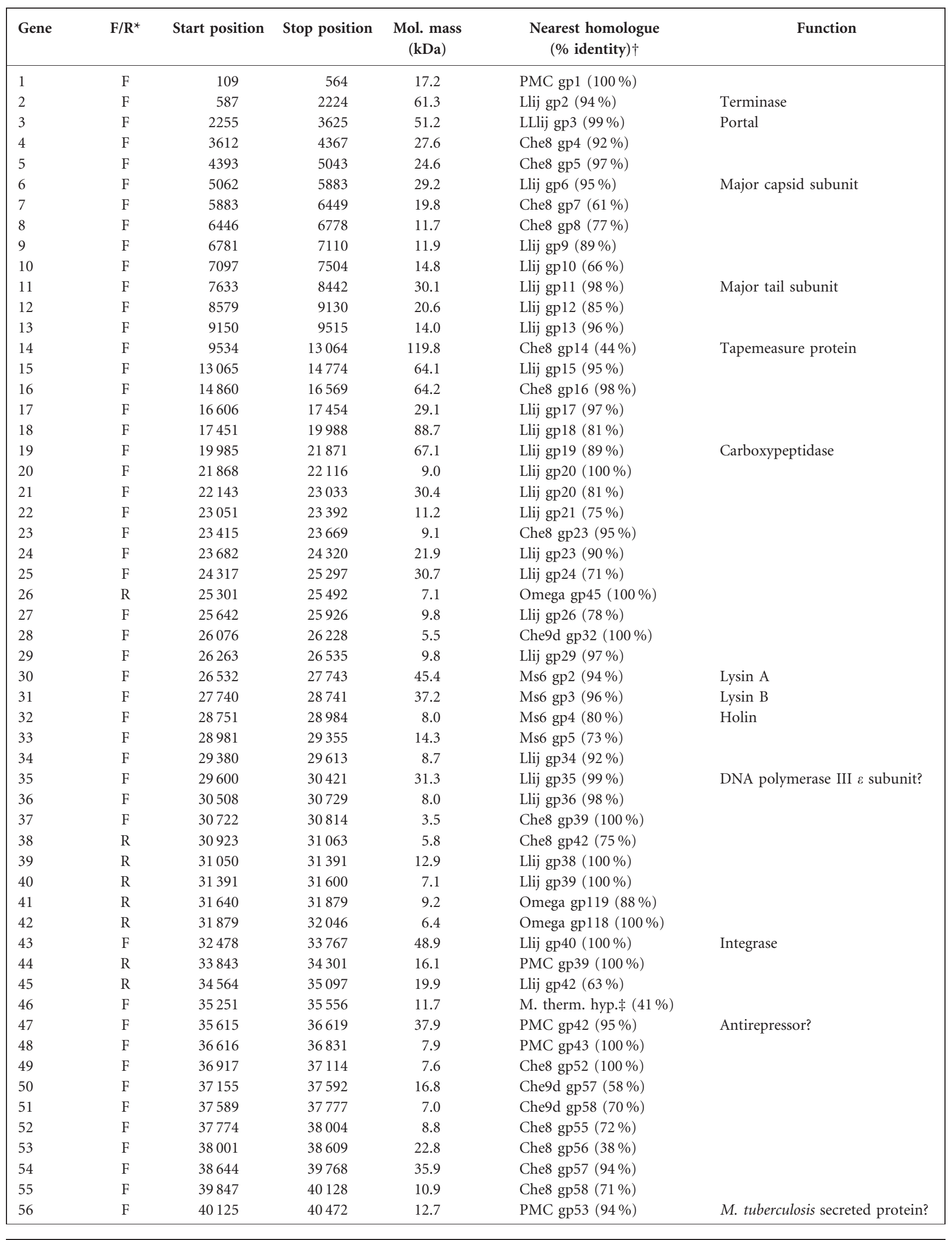


Table 1. cont.

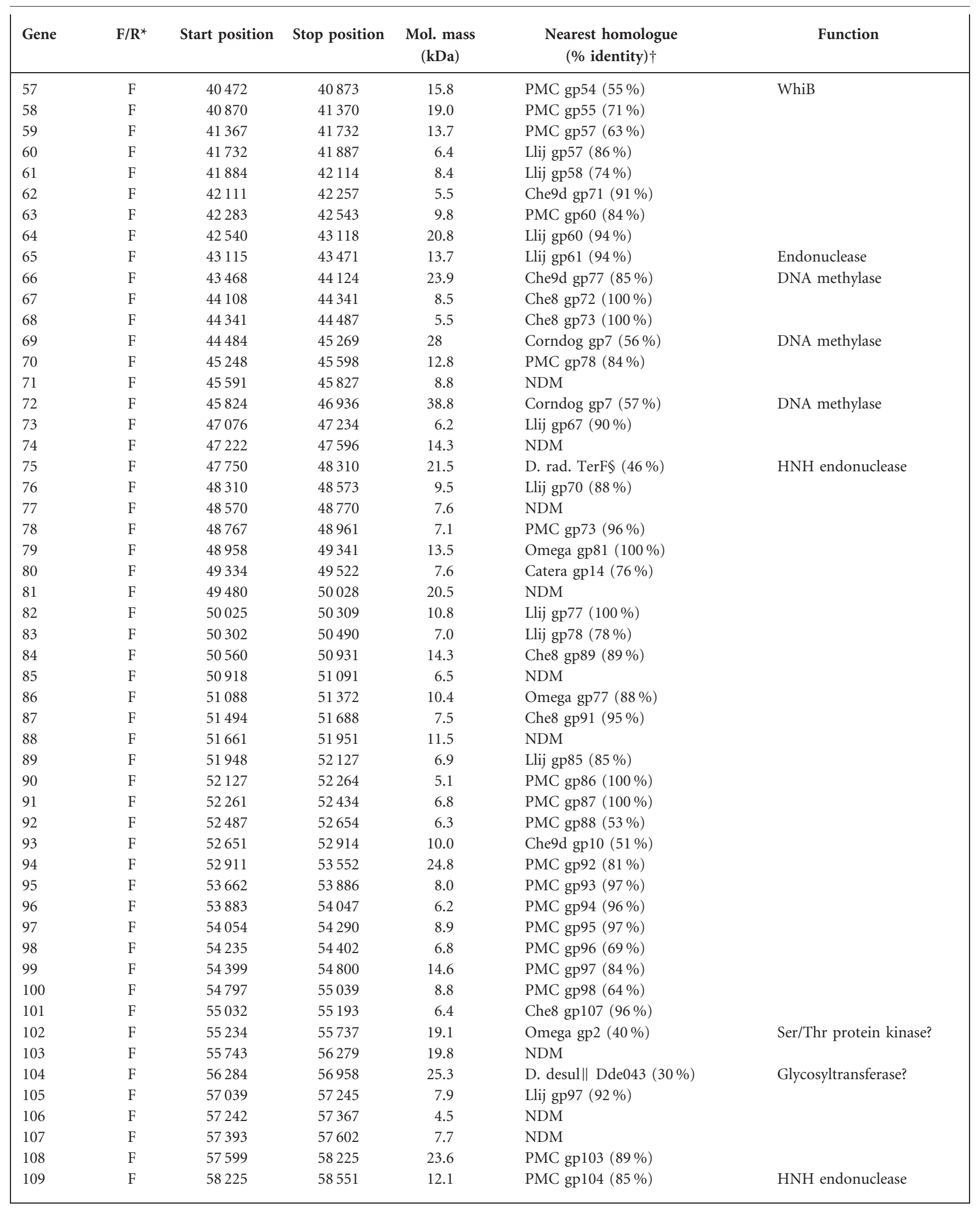


Table 1. cont.

${ }^{\star}$ Direction of transcription, F, forward (leftwards); R, reverse (rightwards).

$\uparrow$ NDM, no database match.

$\$$ M. therm hyp. Mycobacterium thermoresistible hypothetical protein.

$\S D$. rad., Deinococcus radiodurans.

||D. desul Desulfovibrio desulfuricans.

biological consequences for virion particles. We note that a similar single-base deletion in the side tail fibre gene of phage lambda has a specific effect on adsorption to E. coli (Hendrix \& Duda, 1992) and these may thus reflect the types of mutations that fuel the high degree of variation seen among phage tail fibre proteins (Desplats \& Krisch, 2003; Leiman et al., 2006).

\section{The Tweety genomic right arm}

The right arm genes (44-109) are organized distinctly differently from those of phages Che8, Llij and PMC (Fig. 3) and show evident mosaicism, with numerous insertions and deletions, and many genes related to others dispersed throughout other mycobacteriophage genomes. Only few functions of these right arm genes can be predicted, although these include three possible restriction endonucleases (65, 75 and 109) and three probable DNA methylases $(66,69$ and 72$)$. The product of gene 47 is similar to proteins with antirepressor activities, although the immunity functions of Tweety or PMC (which carries a homologue of this protein) have yet to be characterized. We note, however, that gp57 is related to WhiB-family transcriptional regulators, and these are quite common among mycobacteriophage genomes. Tweety also encodes an apparent glycosyl transferase (gp104), a function that has been seen occasionally in other phage genomes, though none of these is a member of the sequence family represented by Tweety gp104. The specific role in the Tweety life cycle is unknown, but since this class of enzymes is associated with modifications of both bacterial cell walls and DNA, it could be involved either in phage exclusion or in protection from restriction. Tweety gp102 has weak sequence similarity to parts of bacterial serine/ threonine protein kinases.

\section{Tweety gp54: a protein with multiple tetrapeptide repeats}

Tweety gp54 is a remarkable protein with high sequence similarity ( $>95 \%$ identity) at both its $\mathrm{N}$ - and C-termini to the corresponding parts of Che 8 gp57 and PMC gp51 (Fig. 4). The first striking aspect of Tweety gene 54 is the presence of a central core of very high mol\% $\mathrm{G}+\mathrm{C}$ that is prominent within a mol\% G $+\mathrm{C}$ scan of the entire Tweety genome (Fig. 4a). Although such a deviation from the average $\mathrm{mol} \% \mathrm{G}+\mathrm{C}$ is often indicative of the introduction of DNA elements by horizontal genetic exchange, in this case this seems unlikely. The segment of high mol\% $\mathrm{G}+\mathrm{C}$ corresponds to an apparent expansion of a $\mathrm{G}+\mathrm{C}$-rich repeated sequence present in all three related proteins (Supplementary Tables S1 and S2). At the nucleotide level the minimum repeat unit is $12 \mathrm{bp}$ long, of which the first six positions (and their encoded alanine residues) are invariant (Supplementary Table S1). Curiously, positions nine and twelve, which correspond to third codon positions in the utilized reading frame, are also invariant, with greater variation occurring at repeat positions seven (34 Gs, 11 Ts, 3 Cs), eight (45 Gs, 3 As), ten (38 As, 10 Ts) and eleven (38 Gs, $10 \mathrm{As}$ ), corresponding to first and second codon positions (Supplementary Table S1). Nevertheless, only two different amino acids are encoded at the fourth residue of the tetrapeptide repeat (serine 38 times, tyrosine 10 times), and three at the third amino acid position (glycine 34 times, tryptophan 11 times, glutamine 3 times) (Supplementary Table S2). This pattern of substitutions within the repeated elements is consistent with selection for variation within this protein.

This repeat sequence is reminiscent of variable region 2 (VR2) in Bordetella phage BMP-1 (Liu et al., 2002, 2004), in which a 24 bp element (which includes a 19 bp repeat followed by one of three possible 5 bp spacers) is repeated 9-20 times, depending on the phage isolate, in gene $b b p 36$; the role of this variable segment is unknown although it does not appear to reflect changes in host tropism (Liu et al., 2004). While the number of the tetrapeptide repeated segments in mycobacteriophages Tweety gp54, Che8 gp57 and PMC gp51 differs (48 in Tweety, 26 in Che8 and 15 in PMC), these do not simply correspond to the variants observed in different BMP-1 isolates, since the encoded amino acid sequence also differs; in all three phages, the first two positions are invariant alanines, but the composition of the last two positions is distinctly different (Supplementary Table S2). The function of these gene products and the utility of this repeat and its variation is not known, although the finding of similar structures in otherwise unrelated mycobacteriophage and Bordetella phages suggests that these may be more widespread throughout phage populations than had been previously recognized. Finally, we note that the entire ORF is absent from mycobacteriophage Llij, even though closely related homologues flanking this gene in Tweety, PMC and Che8 are present (Fig. 3). Presumably, Tweety gp54 is not essential for viral growth, as has been demonstrated for BMP-1 bbp36 (Liu et al., 2004). 

Llij

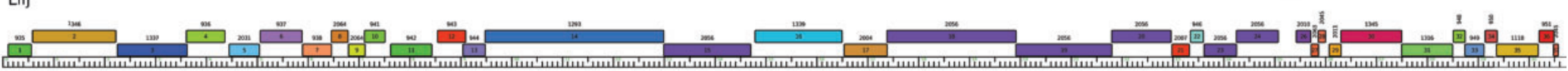
PMC

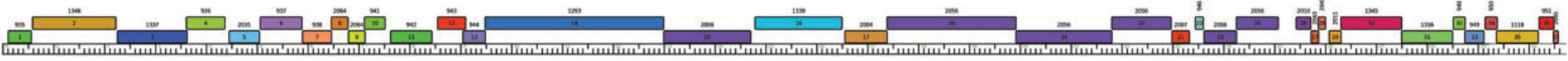
Che9d

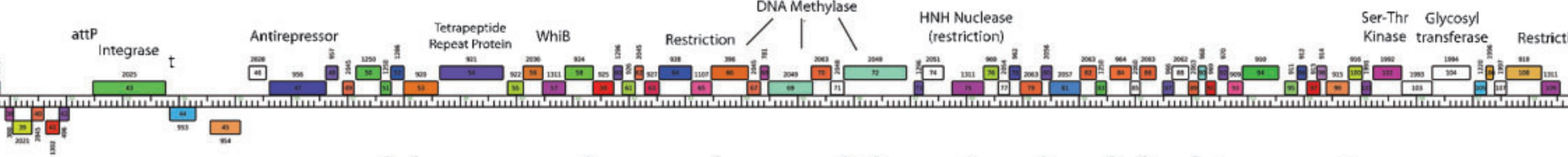

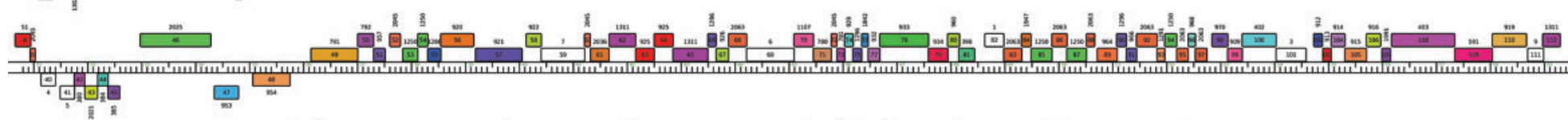

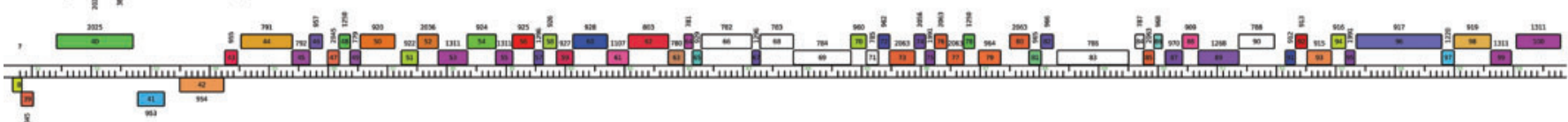

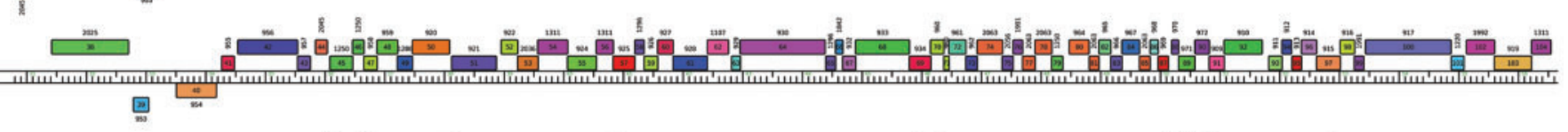

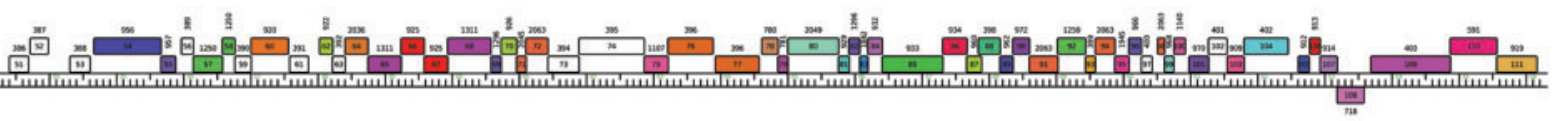

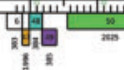

Fig. 3. Map of the Tweety genome and comparison to maps of Che8, Llij, PMC and Che9d. Genomes are represented by horizontal lines with putative genes shown as boxes above (transcribed rightwards) or below (transcribed leftwards) each genome; the number of each gene is shown within each box. The diagonal arrow indicates a programmed translational frameshift between Tweety genes 12 and 13. All genes have been assorted into phamilies (Phams) of related sequences using the computer program 'Phamerator' (S. Cresawn, R. W. Hendrix \& G. F. Hatfull, unpublished data); the phamily number is displayed above each gene and the boxes colour-coordinated accordingly. Note that the Pham numbers differ from those described previously (Hatfull et al., 2006). Putative gene functions are noted. (A larger version of this figure is available as supplementary data with the online version of this paper.) 


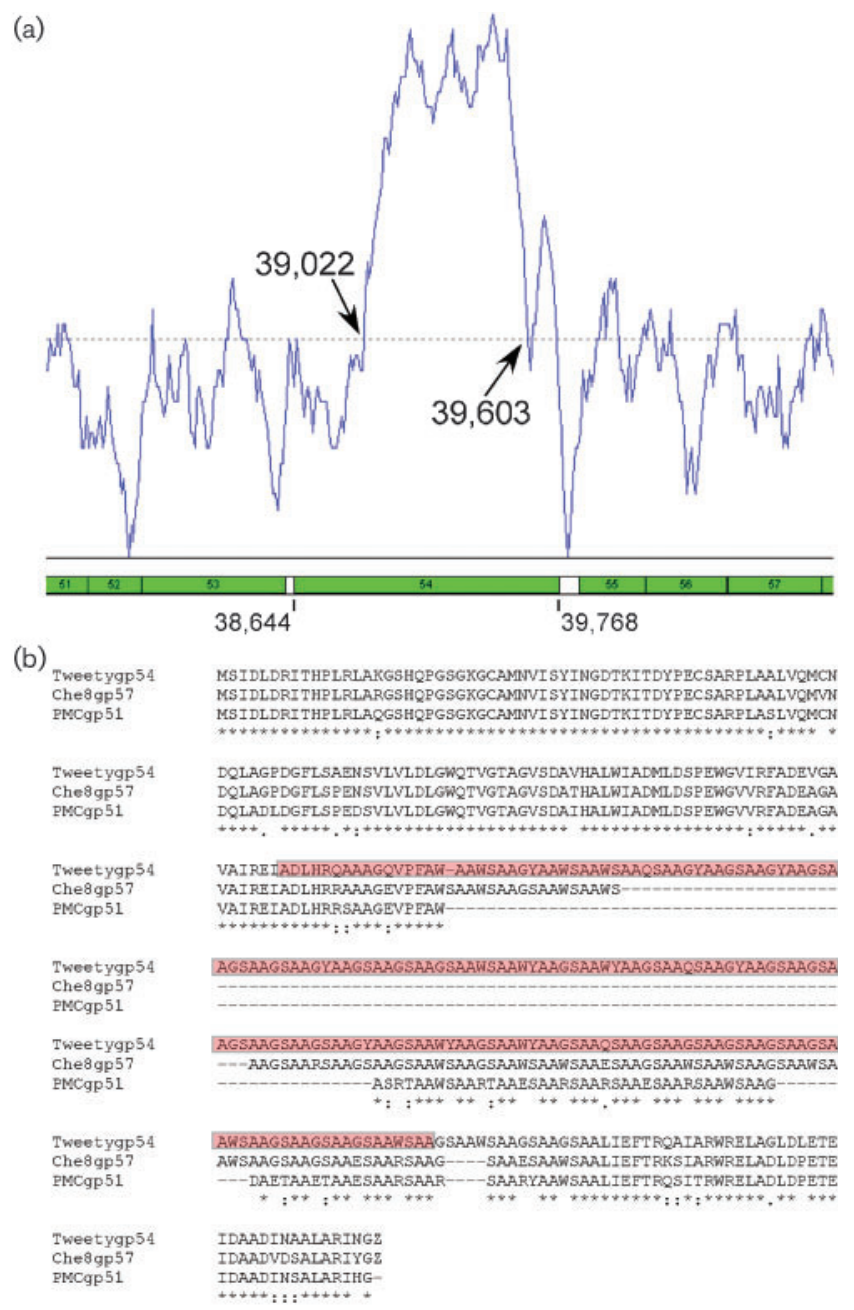

(c) MSIDLDRITHPLRLAKGSHQPGSGKGCAMNVISYINGDTKITDYPECSAR PLAALVQMCNDQLAGPDGFLSAENSVLVLDLGWQTVGTAGVSDAVHALWI ADMLDSPEWGVIRFADEVGAVAIRE IADLHRQAAAGQVPFAWAWISAAGY AAWSIAQSAAGY AAGYAGSAAGS AAGYAMGAAGS AAWSIANAMAGS AAGSAAOSAAGY AAGSAGSAAGS AAGYAAGSAAWY AAWYAAGAAQS AAGSAAGAAGS AA WSMAGSAAGS AAWSIAGSAAWS AAGSAALIEFTRQAIARWRE LAGLDLETEIDAADINAALARINGZ

Fig. 4. An unusual repeated sequence within Tweety gene 54. (a) Plot of $\mathrm{mol} \% \mathrm{G}+\mathrm{C}$ across the Tweety genome, revealing a region of very high mol\% $\mathrm{G}+\mathrm{C}$ within gene 54 . The approximate positions where changes in mol\% $\mathrm{G}+\mathrm{C}$ occur are indicated. (b) Alignment of Tweety gp54, Che8 gp57 and PMC gp51, with amino acid identities shown by asterisks. Conserved substitutions are indicated by colons, and semi-conserved substitutions by periods. The red box indicates the amino acid sequence of Tweety gp54 that corresponds to the segment of high mol\% $\mathrm{G}+\mathrm{C}$ in panel a. (c) Sequence of Tweety gp54 showing the locations of repeated sequences. The repeats can be organized as octapeptide repeats (shown as alternating green and red boxes), or as tetrapeptide repeats (shown as alternating darker- and lightercoloured boxes). Alignments of the sequences of the nucleotide and tetrapeptide repeats are shown in Supplementary Tables S1 and $\mathrm{S} 2$ respectively.

\section{Tweety integration functions}

At the $5^{\prime}$ site of the integrase gene (43) there is a region of approximately $500 \mathrm{bp}$ that lacks protein-coding potential and is a plausible location for the attP site. Comparison of this region with the $M$. smegmatis genome using BLASTN revealed a short segment of sequence identity (45/47 identical base pairs) that overlaps the $3^{\prime}$ end of a host tRNA ${ }^{\text {Lys }}$ gene, a common target for phage integration (Fig. 5a). This indicates that the attP site lies upstream of the Tweety int gene and that Tweety integrates at an $a t t B$ site located at coordinates 4847939-4847986 in the M. smegmatis genome. This arrangement also suggests that integration of Tweety results in reconstruction of a hybrid but functional tRNA gene of which the sequence $3^{\prime}$ to the extreme $5^{\prime}$-side of the anticodon stem is phage-derived (Fig. 5b). Interestingly, the two base differences between Tweety and the M. smegmatis genome correspond to the innermost-paired bases in the T $\psi \mathrm{C}$ loop of the tRNA (Fig. 5b). Comparison with other mycobacterial genomes shows that this tRNA and the putative attB sites are conserved in M. tuberculosis, M. bovis, Mycobacterium leprae and Mycobacterium avium. We also note that mycobacteriophages Che8, Llij and PMC contain near-identical integrases and putative attP sites, and probably integrate at the same chromosomal location. Che9d has a closely related integrase (39\% amino acid sequence identity) but a different putative attP site that we predict recombines at a tRNA ${ }^{\text {Met }}$ gene (see below).

\section{Tweety-based integration-proficient plasmid vectors}

The putative attB site is at a distinct location from those previously described for phages L5, Ms6 and Bxb1. We therefore reasoned that integration-proficient vectors derived from phage Tweety would integrate independently from those derived from other phages and could thus be used in conjunction with them without interference. Furthermore, the conservation of the host tRNA ${ }^{\text {Lys }}$ gene provides a potentially broad host range for integrating plasmids. To construct such vectors, a $1.7 \mathrm{kbp}$ segment of the Tweety genome corresponding to the int gene and $\sim 400$ bp of upstream sequences containing the putative attP site were PCR amplified and cloned into a plasmid vector containing a kanamycin-resistance gene that cannot replicate in mycobacteria (Fig. 5c). The two plasmids with the attP-int segment in either orientation (pTTP1A and pTTP1B) were electroporated into M. smegmatis and the numbers of kanamycin-resistant transformants determined (Table 2); both plasmids efficiently transformed $M$. smegmatis, yielding approximately $10^{5}$ transformants per $\mu \mathrm{g}$ DNA. PCR analysis showed that every transformant tested derived from integration of the plasmid sequences at the predicted $a t t B$ site (data not shown).

To test whether these Tweety integration-proficient vectors are fully compatible with the previously described L5 integration-proficient vectors, we performed co-electroporations with either pTTP1A or pTTP1B DNA and pJV39, 
(a)

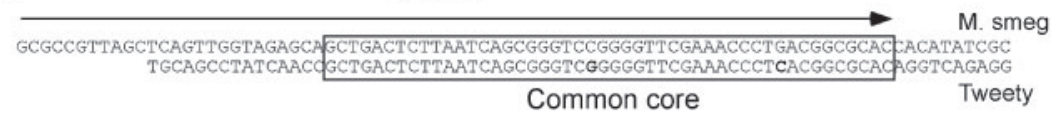

(b)

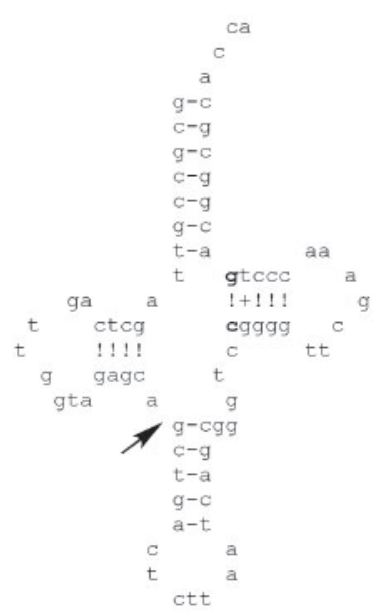

(c)

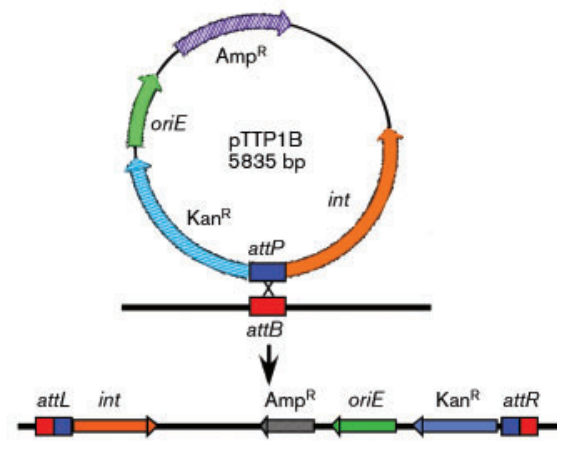

Fig. 5. Integration of Tweety into the $M$. smegmatis genome. (a) Sequence alignment of a segment of the Tweety genome immediately upstream of the int gene (43) (coordinates 32362-32 290) with part of the $M$. smegmatis genome (coordinates $4847908-$ 4847 991) reveals a common core sequence (boxed); two non-identical positions are shown in bold type. The position of the M. smegmatis tRNA ${ }^{\text {Lys }}$ gene (Msmeg 4746) is indicated by the arrow. (b) Structure of tRNA ${ }^{\text {Lys }}$, with an arrow indicating the position corresponding to the $5^{\prime}$ position of the common core. Positions that change following Tweety integration are shown in bold. (c) Organization of integrationproficient plasmid pTT1B and its integration into the $M$. smegmatis chromosome. an L5 integration vector conferring hygromycin resistance. Co-transformants were readily recovered, indicating that these plasmid integration systems do not interfere with each other (Table 2). We also prepared electrocompetent cells carrying an L5-integration-proficient plasmid vector and showed that pTTP1A efficiently transforms this strain (data not shown). A similar series of experiments were performed using BCG with similar outcomes although the overall transformation frequencies were somewhat lower (Table 2).

The stability of integration-proficient vectors is dependent on the absence of the phage-encoded excise gene. Plasmids pTTP1A and pTTP1B contain no other annotated ORFs other than the integrase gene, so we presume that the putative excise gene is absent. We have not been able to identify any putative excise gene by sequence analysis, although the best candidate is gene 44, not only because it is adjacent to int, but also because there are related copies in phages Che8, PMC and LLij that encode identical integrases (Fig. 3). To test for plasmid stability we grew $M$. smegmatis transformants in the absence of antibiotic selection for approximately 35 generations and then determined the proportion of recovered colonies that had lost the plasmid drug-resistance gene. Under these conditions, we observed that approximately $15 \%$ of cells had lost an L5-derived integrated plasmid ( $\mathrm{pMH} 94$ ) whereas only $3.3 \%$ and $7.4 \%$ had lost plasmids pTTP1A and pTTP1B respectively. As noted previously for L5 vectors, the stability of these Tweety vectors could probably be further

Table 2. Transformation of M. smegmatis and BCG by Tweety and L5 integration-proficient vectors

\begin{tabular}{|c|c|c|c|c|c|}
\hline \multirow[t]{2}{*}{$\operatorname{Plasmid}(s)$} & \multirow[t]{2}{*}{ Features } & \multirow[t]{2}{*}{ Host } & \multicolumn{3}{|c|}{ Transformants [c.f.u. $(\mu \mathrm{g} \text { DNA })^{-1}$ ] } \\
\hline & & & $\operatorname{Kan}^{R}$ & $\mathrm{Hyg}^{\mathrm{R}}$ & $\operatorname{Kan}^{\mathrm{R}} / \mathrm{Hyg}^{\mathrm{R}}$ \\
\hline \multirow[t]{2}{*}{ pTTP1A } & \multirow[t]{2}{*}{ Tweety attP-int; $\mathrm{Kan}^{\mathrm{R}}$} & M. smegmatis & $2 \times 10^{5}$ & - & - \\
\hline & & BCG & $4 \times 10^{5}$ & - & - \\
\hline \multirow[t]{2}{*}{ pTTP1B } & \multirow[t]{2}{*}{ Tweety attP-int; $\mathrm{Kan}^{\mathrm{R}}$} & M. smegmatis & $1 \times 10^{5}$ & - & - \\
\hline & & BCG & $3 \times 10^{5}$ & - & - \\
\hline \multirow[t]{2}{*}{ pMH94 } & \multirow[t]{2}{*}{ L5 attP-int; $\mathrm{Kan}^{\mathrm{R}}$} & M. smegmatis & $2 \times 10^{5}$ & - & - \\
\hline & & BCG & $2 \times 10^{5}$ & - & - \\
\hline \multirow[t]{2}{*}{ pJV39 } & \multirow[t]{2}{*}{ L5 attP-int; $\mathrm{Hyg}^{\mathrm{R}}$} & M. smegmatis & - & $3 \times 10^{5}$ & - \\
\hline & & BCG & - & $4 \times 10^{5}$ & - \\
\hline \multirow[t]{2}{*}{ pTTP1A + pJV39 } & \multirow[t]{2}{*}{ Tweety attP-int; $\mathrm{Kan}^{\mathrm{R}}+\mathrm{L} 5$ attP-int; $\mathrm{Hyg}^{\mathrm{R}}$} & M. smegmatis & $2 \times 10^{4}$ & $6 \times 10^{4}$ & $2 \times 10^{3}$ \\
\hline & & BCG & $2 \times 10^{4}$ & $1 \times 10^{4}$ & $2 \times 10^{3}$ \\
\hline \multirow[t]{2}{*}{ pTTP1B + pJV39 } & \multirow[t]{2}{*}{ Tweety attP-int; $\operatorname{Kan}^{\mathrm{R}}+\mathrm{L} 5$ attP-int; $\mathrm{Hyg}^{\mathrm{R}}$} & M. smegmatis & $2 \times 10^{4}$ & $4 \times 10^{4}$ & $8 \times 10^{3}$ \\
\hline & & BCG & $2 \times 10^{4}$ & $7 \times 10^{3}$ & $2 \times 10^{3}$ \\
\hline
\end{tabular}


increased by using a transient integrase-expression system (Hatfull, 2004, 2006).

\section{DISCUSSION}

We have presented here the genome of mycobacteriophage Tweety, a new mycobacteriophage with several interesting and novel features, and its exploitation for the development of integration-proficient vectors that are compatible with those described previously. The Tweety genome is most closely related to those of Che8, PMC and Llij (Hatfull et al., 2006) (Fig. 2), and this close similarity allows more fine-scale conclusions about evolutionary changes than are available from comparisons among more distantly related phages. Only a few of the Tweety gene functions can be readily predicted, although these include several possible restriction endonucleases and several DNA methylases. However, these do not form well-defined restriction-modification cassettes, and combinations of these are not well conserved in the other closely related mycobacteriophages (Fig. 3). For example, homologues of Tweety gp65 are found in Che8, Llij, PMC and Che9d and there are more distant relatives in mycobacteriophages Cjw1 and Wildcat (Hatfull et al., 2006). However, none of these have a closely linked DNA modification function that can be readily recognized.

The presence of a gene encoding a putative family 2 glycosyltransferase (gp104) in the Tweety genome is intriguing since, to our knowledge, this is the first finding of a member of this sequence family of glycosyltransferases in any phage genome. Similar enzymes have been shown previously to be involved in sugar modifications of bacterial cell walls, and gp104 could play a role in phage exclusion similar to the role proposed for the glucosyltransferase in phage SfV (Bastin et al., 1997); however, it is also possible that Tweety gp104 could be involved in DNA modification. Phage T4 and its close relatives encode two glycosyltransferases, and these have long been known to add glucose to hydroxymethyl cytosine residues in phage DNA. If the Tweety enzyme also adds sugars to DNA, this would be an example of analogous but not homologous proteins carrying out the same function in different phages. Other examples include phage lysins, integrases and head-maturation proteases. There do not appear to be any closely related homologues of Tweety gene 104 in any other sequenced mycobacterial genome, and it is therefore unclear from where this gene was acquired. We note that the gene immediately upstream, 103, has no identifiable homologues in other phage genomes or elsewhere.

Tweety gp54 is unusual with respect to the repeated sequence within the ORF that significantly expands the length of the gene relative to its homologues in phages Che8 (gp57) and PMC (gp51). While the functions of these genes are still unknown, these structures are interesting in their organizational similarity to the VR2 region of Bordetella phage BMP-1. The BMP-1 bbp36 gene that contains VR2 is not essential for phage growth, and we note that Llij does not contain a homologue of Tweety gp54 even though similar flanking genes are present, suggesting that it is not essential for mycobacteriophage growth either. Repeats similar to those in Tweety gp54 are commonly associated with intrinsically unstructured proteins (Tompa, 2003).

The development of integration-proficient vectors with site specificities distinct from those developed previously will provide important tools for constructing recombinant mycobacterial strains. The need for such vectors is illustrated by the development of secondary applications for those derived from phages Ms6 and L5 (Saviola \& Bishai, 2004; Vultos et al., 2006), in which either secondary attB sites have been introduced or specificities have been altered mutationally, albeit with significant loss of efficiency (Vultos et al., 2006). The Tweety integration vectors not only transform both fast- and slow-growing strains efficiently, but do so in a manner that is fully compatible with integration vectors derived from L5 (Table 2) and Bxb1 (data not shown); it is likely that they are also compatible with Ms6-derived vectors. The Tweety vectors are also maintained with reasonable stability in the

Table 3. Mycobacteriophage integration systems and putative integration sites

\begin{tabular}{|c|c|c|c|c|}
\hline Phage(s) & Integrase type & $a t t B$ location & $\begin{array}{l}\text { M. smegmatis } \\
\text { coordinates }\end{array}$ & $\begin{array}{c}\text { M. tuberculosis? } \\
\text { (bp identity) }\end{array}$ \\
\hline L5, D29, Che12 & Tyr & $\mathrm{tRNA}^{\text {Gly }}$ & $4764522-4764564$ & Yes $(42 / 43)$ \\
\hline Tweety, Che8, Llij, PMC & Tyr & $\mathrm{tRNA}^{\text {Lys }}$ & $4847934-4847981$ & Yes $(46 / 48)$ \\
\hline Ms6 & Tyr & $\mathrm{tRNA}^{\mathrm{Ala}}$ & $2213189-2213214$ & Yes $(26 / 26)$ \\
\hline Che9d & Tyr & $\mathrm{tRNA}^{\mathrm{Met}}$ & $4532823-4532867$ & Yes? (42/45) \\
\hline Halo & Tyr & $\mathrm{tRNA}^{\mathrm{Arg}}$ & $6410365-6410399$ & No \\
\hline Omega & Tyr & $\mathrm{tRNA}^{\mathrm{Leu}}$ & $3328692-3328735$ & Yes? (39/42) \\
\hline Che9c & Tyr & $\mathrm{tRNA}^{\mathrm{Tyr}}$ & $1228421-1228480$ & Yes? (53/57) \\
\hline CJW1, 244 & Tyr & $?$ & $?$ & $?$ \\
\hline Bxb1, U2, Bethlehem & Ser & groEL1 & & No \\
\hline Bxz2 & Ser & Msmeg_5156 & $5259344-5259347$ & No \\
\hline
\end{tabular}


absence of drug selection, and somewhat more so than the L5-derived vectors. We have not yet been able to identify the Tweety recombination-directionality factor by sequence comparisons, which is perhaps not surprising given the high sequence divergence of these proteins (Lewis \& Hatfull, 2001), although Tweety gp44 remains the best candidate for this function.

While integrase genes can be readily identified in phage genomes, the locations of the attP sites require somewhat closer examination. The putative location of Tweety attP was indicated by sequence comparison with the $M$. smegmatis genome, and is facilitated by the use of an attB site that overlaps a host tRNA gene which is reconstructed following integration. Thus finding a long common core (40 bases or more) that overlaps a host tRNA gene is strongly predictive of the attB site location. We have extended this approach to identify potential att $B$ sites of other mycobacteriophage integrases in order to identify those that are the best candidates for development of additional integration-proficient vectors with new specificities (Table 3). Using this approach, we predict that phages Che $9 \mathrm{~d}$, Che9c, Halo and Omega integrate at tRNA $^{\text {Met }}$, tRNA ${ }^{\text {Tyr }}$, tRNA ${ }^{\text {Arg }}$ and tRNA ${ }^{\text {Leu }}$ genes respectively, using attB sites that are distinct from those of L5, Ms6 and Tweety; three of these phages have conserved attB sites in $M$. tuberculosis (Table 3), suggesting that these could be potential broad-host-range integration systems. Interestingly, the Halo integration site is similar to that suggested previously for beta family phages of the Corynebacteria (Cianciotto et al., 1990). This strategy is not applicable for those phages that use serine integrases, although we have identified the attB site for the Bxz2 serine integrase, which is located within the Msmeg_5156 ORF, using experimental approaches (Table 3). The Bxz2 attP and its attB sites share only a $4 \mathrm{bp}$ common core and thus could not simply be identified bioinformatically.

In summary, the genomic analysis of mycobacteriophage Tweety and the development of new integration-proficient vectors further illustrate the general utility of mycobacteriophage studies for mycobacterial genetics. Most of the Tweety genomic functions have yet to be explored or exploited, but this phage promises to have potential utility for understanding other important aspects of mycobacterial and bacteriophage biology and evolution.

\section{ACKNOWLEDGEMENTS}

This work was supported by a grant to the University of Pittsburgh from the Howard Hughes Medical Institute (HHMI) in support of G. F. H. under HHMI's Professors program, and by grants from NIH to R.W.H. (GM5197508), and to G. F.H (AI28927). We thank Julia van Kessel for plasmids pJV39 and pJV44, Jennifer Houtz and Alexis Smith for assistance with sequencing, Molly Scanlon, Amy Vogelsberger and other lab members for excellent technical support, Marcus Bell for information regarding the mapping of the Bxz2 integration site, Andrew Sachs and John Hempel for sequence determination of the Che8 major capsid subunit, Tom Harper for help with electron microscopy, Steven Cresawn for discussion and map generation, and Craig Peebles for helpful discussions and preparation of Supplementary Fig. S2.

\section{REFERENCES}

Banerjee, A., Dubnau, E., Quemard, A., Balasubramanian, V., Um, K. S., Wilson, T., Collins, D., de Lisle, G. \& Jacobs, W. R., Jr (1994). inhA, a gene encoding a target for isoniazid and ethionamide in Mycobacterium tuberculosis. Science 263, 227-230.

Barsom, E. K. \& Hatfull, G. F. (1996). Characterization of Mycobacterium smegmatis gene that confers resistance to phages L5 and D29 when overexpressed. Mol Microbiol 21, 159-170.

Bastin, D. A., Lord, A. \& Verma, N. K. (1997). Cloning and analysis of the glucosyl transferase gene encoding type I antigen in Shigella flexneri. FEMS Microbiol Lett 156, 133-139.

Bibb, L. A. \& Hatfull, G. F. (2002). Integration and excision of the Mycobacterium tuberculosis prophage-like element, $\phi$ Rv1. Mol Microbiol 45, 1515-1526.

Bibb, L. A., Hancox, M. I. \& Hatfull, G. F. (2005). Integration and excision by the large serine recombinase $\phi$ Rv1 integrase. Mol Microbiol 55, 1896-1910.

Brown, K. L., Sarkis, G. J., Wadsworth, C. \& Hatfull, G. F. (1997). Transcriptional silencing by the mycobacteriophage L5 repressor. EMBO J 16, 5914-5921.

Cianciotto, N., Serwold-Davis, T., Groman, N., Ratti, G. \& Rappuoli, R. (1990). DNA sequence homology between attB-related sites of Corynebacterium diphtheriae, Corynebacterium ulcerans, Corynebacterium glutamicum, and the attP site of $\gamma$-corynephage. FEMS Microbiol Lett 54, 299-301.

Desplats, C. \& Krisch, H. M. (2003). The diversity and evolution of the T4-type bacteriophages. Res Microbiol 154, 259-267.

Donnelly-Wu, M. K., Jacobs, W. R., Jr \& Hatfull, G. F. (1993). Superinfection immunity of mycobacteriophage L5: applications for genetic transformation of mycobacteria. Mol Microbiol 7, 407-417.

Ford, M. E., Sarkis, G. J., Belanger, A. E., Hendrix, R. W. \& Hatfull, G. F. (1998). Genome structure of mycobacteriophage D29: implications for phage evolution. J Mol Biol 279, 143-164.

Freitas-Vieira, A., Anes, E. \& Moniz-Pereira, J. (1998). The sitespecific recombination locus of mycobacteriophage Ms6 determines DNA integration at the tRNA(Ala) gene of Mycobacterium spp. Microbiology 144, 3397-3406.

Hatfull, G. F. (2004). Mycobacteriophages and tuberculosis. In Tuberculosis, pp. 203-218. Edited by K. Eisenach, S. T. Cole, W. R. Jacobs, Jr \& D. McMurray. Washington, DC: American Society for Microbiology.

Hatfull, G. F. (2006). Mycobacteriophages. In The Bacteriophages, pp. 602-620. Edited by R. Calendar. New York, NY: Oxford University Press.

Hatfull, G. F. \& Sarkis, G. J. (1993). DNA sequence, structure and gene expression of mycobacteriophage L5: a phage system for mycobacterial genetics. Mol Microbiol 7, 395-405.

Hatfull, G. F., Pedulla, M. L., Jacobs-Sera, D., Cichon, P. M., Foley, A., Ford, M. E., Gonda, R. M., Houtz, J. M., Hryckowian, A. J. \& other authors (2006). Exploring the mycobacteriophage metaproteome: phage genomics as an educational platform. PLoS Genetics 2, e92.

Hendrix, R. W. \& Duda, R. L. (1992). Bacteriophage lambda PaPa: not the mother of all lambda phages. Science 258, 1145-1148.

Jacobs, W. R., Jr, Kalpana, G. V., Cirillo, J. D., Pascopella, L., Snapper, S. B., Udani, R. A., Jones, W., Barletta, R. G. \& Bloom, B. R. (1991). Genetic systems for mycobacteria. Methods Enzymol 204, 537-555. 
Jain, S. \& Hatfull, G. F. (2000). Transcriptional regulation and immunity in mycobacteriophage Bxb1. Mol Microbiol 38, 971-985.

Katsura, I. \& Hendrix, R. W. (1984). Length determination in bacteriophage lambda tails. Cell 39, 691-698.

Kim, A. I., Ghosh, P., Aaron, M. A., Bibb, L. A., Jain, S. \& Hatfull, G. F. (2003). Mycobacteriophage Bxbl integrates into the Mycobacterium smegmatis groEL1 gene. Mol Microbiol 50, 463-473.

Lee, M. H., Pascopella, L., Jacobs, W. R., Jr \& Hatfull, G. F. (1991). Site-specific integration of mycobacteriophage L5: integrationproficient vectors for Mycobacterium smegmatis, Mycobacterium tuberculosis, and bacille Calmette-Guerin. Proc Natl Acad Sci U S A 88, 3111-3115.

Leiman, P. G., Shneider, M. M., Mesyanzhinov, V. V. \& Rossmann, M. G. (2006). Evolution of bacteriophage tails: structure of T4 gene product 10. J Mol Biol 358, 912-921.

Lewis, J. A. \& Hatfull, G. F. (2000). Identification and characterization of mycobacteriophage L5 excisionase. Mol Microbiol 35, 350-360.

Lewis, J. A. \& Hatfull, G. F. (2001). Control of directionality in integrase-mediated recombination: examination of recombination directionality factors (RDFs) including Xis and Cox proteins. Nucleic Acids Res 29, 2205-2216.

Liu, M., Deora, R., Doulatov, S. R., Gingery, M., Eiserling, F. A., Preston, A., Maskell, D. J., Simons, R. W., Cotter, P. A. \& other authors (2002). Reverse transcriptase-mediated tropism switching in Bordetella bacteriophage. Science 295, 2091-2094.

Liu, M., Gingery, M., Doulatov, S. R., Liu, Y., Hodes, A., Baker, S., Davis, P., Simmonds, M., Churcher, C. \& other authors (2004). Genomic and genetic analysis of Bordetella bacteriophages encoding reverse transcriptase-mediated tropism-switching cassettes. J Bacteriol 186, 1503-1517.

Ojha, A., Anand, M., Bhatt, A., Kremer, L., Jacobs, W. R., Jr \& Hatfull, G. F. (2005). GroEL1: a dedicated chaperone involved in mycolic acid biosynthesis during biofilm formation in mycobacteria. Cell 123 , 861-873.

Parish, T., Lewis, J. \& Stoker, N. G. (2001). Use of the mycobacteriophage L5 excisionase in Mycobacterium tuberculosis to demonstrate gene essentiality. Tuberculosis (Edinb) 81, 359-364.

Pashley, C. A. \& Parish, T. (2003). Efficient switching of mycobacteriophage L5-based integrating plasmids in Mycobacterium tuberculosis. FEMS Microbiol Lett 229, 211-215.

Pedulla, M. L., Ford, M. E., Houtz, J. M., Karthikeyan, T., Wadsworth, C., Lewis, J. A., Jacobs-Sera, D., Falbo, J., Gross, J. \& other authors
(2003). Origins of highly mosaic mycobacteriophage genomes. Cell $113,171-182$.

Peña, C. E., Lee, M. H., Pedulla, M. L. \& Hatfull, G. F. (1997). Characterization of the mycobacteriophage L5 attachment site, attP. $J$ Mol Biol 266, 76-92.

Sambrook, J., Fritsch, E. F. \& Maniatis, T. (1989). Molecular Cloning: a Laboratory Manual. Cold Spring Harbor, NY: Cold Spring Harbor Laboratory Press.

Sarkis, G. J. \& Hatfull, G. F. (1998). Mycobacteriophages. Methods Mol Biol 101, 145-173.

Saviola, B. \& Bishai, W. R. (2004). Method to integrate multiple plasmids into the mycobacterial chromosome. Nucleic Acids Res 32, e11.

Snapper, S. B., Melton, R. E., Mustafa, S., Kieser, T. \& Jacobs, W. R., $\mathrm{Jr}$ (1990). Isolation and characterization of efficient plasmid transformation mutants of Mycobacterium smegmatis. Mol Microbiol 4, 1911-1919.

Sonnhammer, E. L. \& Durbin, R. (1995). A dot-matrix program with dynamic threshold control suited for genomic DNA and protein sequence analysis. Gene 167, GC1-GC10.

Springer, B., Sander, P., Sedlacek, L., Ellrott, K. \& Bottger, E. C. (2001). Instability and site-specific excision of integration-proficient mycobacteriophage L5 plasmids: development of stably maintained integrative vectors. Int J Med Microbiol 290, 669-675.

Stover, C. K., de la Cruz, V. F., Fuerst, T. R., Burlein, J. E., Benson, L. A., Bennett, L. T., Bansal, G. P., Young, J. F., Lee, M. H. \& other authors (1991). New use of BCG for recombinant vaccines. Nature 351, 456-460.

Tompa, P. (2003). Intrinsically unstructured proteins evolve by repeat expansion. Bioessays 25, 847-855.

van Kessel, J. C. \& Hatfull, G. F. (2007). Recombineering in Mycobacterium tuberculosis. Nat Methods 4, 147-152.

Vultos, T. D., Mederle, I., Abadie, V., Pimentel, M., Moniz-Pereira, J., Gicquel, B., Reyrat, J. M. \& Winter, N. (2006). Modification of the mycobacteriophage Ms6 attP core allows the integration of multiple vectors into different tRNA ${ }^{\text {ala }}$ T-loops in slow- and fast-growing mycobacteria. BMC Mol Biol 7, 47.

Xu, J., Hendrix, R. W. \& Duda, R. L. (2004). Conserved translational frameshift in dsDNA bacteriophage tail assembly genes. Mol Cell 16, $11-21$.

Edited by: S. V. Gordon 